\title{
Evaluation of the TCS TA system for the detection of anti-rubella antibodies
}

\author{
Olivia Arpino, Annalisa Cianflone, Maria Teresa Manco, Alessia Paganini, Massimo De Paschale, \\ Carlo Agrappi, Paola Mirri, Pierangelo Clerici
}

Microbiology Unit, Legnano Hospital, ASST Ovest Milanese, Legnano, Italy

\section{Summary}

Background and aims: The aim of the present study was to evaluate the new Technogenetics TGS TA system for detecting antirubella IgG and IgM antibodies and IgG avidity. TGS TA system was compared with our routinely used system, LIAISON XL, for the detection of IgG and IgM antibodies. Only in positive IgM samples (retrospective study), TGS TA system was compared to an ELFA IgM test and with an ELISA test for the IgG avidity (if existent) .

Materials and methods: Two hundred and seventy six sera samples from women were examined with TGS TA system and divided in 3 groups according to IgG and IgM screening LIAISON XL tests: 112 were of childbearing age and non-immune women (Group 1), 106 were pregnant with past infection or vaccinated (Group 2) and 49 were pregnant with positive or equivocal IgM (Group 3).

Results: The overall concordance of the IgG results between LIAISON XL and TGS TA was $93.3 \%$ : 86.6\% in Group 1, 97.2\% in Group 2 and $100 \%$ in Group 3.

The overall concordance of the IgM results between LIAISON XL and TGS TA was 89.0\%: 100\% in Group 1, 100\% in Group 2 and $35.6 \%$ in Group 3. In Group 3, the concordance between the results of the IgG avidity with the ELISA and TGS TA tests was $85.7 \%$. Comparing the clinical diagnosis obtained with our protocol and that of the TGS TA system, the overall concordance was $97.4 \%$ : $86.6 \%$ in Group 1, 97.2\% in Group 2 and $85.7 \%$ in Group 3.

\footnotetext{
Correspondence: Olivia Arpino, Microbiology Unit, Legnano Hospital, ASST Ovest Milanese, via Papa Giovanni Paolo II, Legnano (MI), Italy. Tel.: +39.0331.449319.

E-mail: olivia.arpino86@gmail.com
}

Key words: Rubella; Pregnancy; IgM; IgG; Avidity.

Contributions: the authors contributed equally.

Conflict of interest: the authors declare no potential conflict of interest.

Received for publication: 18 January 2017.

Revision received: 13 March 2017.

Accepted for publication: 15 March 2017.

CC Copyright O. Arpino et al., 2017

Licensee PAGEPress, Italy

Microbiologia Medica 2017; 32:6582

doi: $10.4081 / \mathrm{mm} .2017 .6582$

This article is distributed under the terms of the Creative Commons Attribution Noncommercial License (by-nc 4.0) which permits any noncommercial use, distribution, and reproduction in any medium, provided the original author(s) and source are credited.
Conclusions: TGA TS system shows to be a valuable tool with overall good clinical correlation and able to clearly identify nonspecific subjects, those with a non-recent infection or those who are vaccinated. The TGS TA test also seems to be especially sensitive in indicating vaccinated subjects with low IgG levels as immune.

\section{Introduction}

Rubella is a generally benign disease in childhood but, if contracted during pregnancy, it might cause miscarriage or serious malformations of the foetus (10). The risk of foetal damage is highest in the first three months of pregnancy then diminishes rapidly after the 12th week to rare cases of deafness in infections occurring in the 17-18th week $(2,4)$. Various European states started vaccination campaigns reflecting two main strategies (or a combination of the two) to prevent the onset of congenital infection (CRS). Those strategies are a selective vaccination for adolescent women and/or of childbearing age or a universal vaccination of children $(1,7,12)$. Strategies focusing on pregnant women have also been introduced such as screening to detect anti-rubella antibodies as early as possible with, in case of negative results, a repetition every 4-6 weeks until at least the 5 th month of age, and the vaccination of susceptible women in the post-partum period $(3,8,11)$. Several analytical systems, with different automation, are currently available for antibody search such as Enzyme-Linked Immunosorbent Assay (ELISA), Chemiluminescence Immunoassay (CLIA) and Enzyme Linked Fluorescent Assay (ELFA) able, depending on the instrument used, to process a great number of samples in a short time. The commercial tests available generally have excellent sensitivity and specificity levels, and the slight differences in the results obtained with the different tests could depend on the differences in formulations and in the use of natural, recombinant or synthetic antigens. The extent of these differences must be known. We compared the data obtained with Technogenetics' TGS TA system for detecting anti-rubella IgG and $\operatorname{IgM}$ antibodies and $\operatorname{IgG}$ avidity with the results obtained with our protocol which first of all sets out detection of IgG and IgM antibodies with the DiaSorin LIAISON XL system, in order to assess the differences that may arise using different systems. In our protocol, in case of $\operatorname{IgM}$ positivity, the samples are confirmed using an ELFA test and the IgG avidity is determined (if existent) with an ELISA test.

\section{Materials and Methods}

Overall we examined 276 serum samples, divided into the groups below: 
Group 1: 112 samples from non-immune women of childbearing age;

Group 2: 106 samples from pregnant women with past infection or vaccinated;

Group 3: 49 samples from pregnant women with positive or equivocal IgM.

We selected samples from non-immune women of childbearing age (Group 1) and pregnant women with past infection or vaccinated (Group 2) from our serological routine conducted from 30 June-31 July 2016 (perspective study).

We selected samples from pregnant women with positive or equivocal IgM to the LIAISON XL (Group 3) from our serum bank (retrospective study).

Routinely, samples are tested using chemiluminescence LIAISON XL system for IgG and IgM (LIAISON Rubella IgG, IgM, DiaSorin, Saluggia, Italy). In positive subjects for IgM, samples are tested using an ELFA test (VIDAS RUB IgM, BioMérieux, Marcy l'Etoile, France). The avidity of the IgG (if present) is determined with an ELISA test (Enzywell Rubella IgG Avidity, DIESSE, Monteriggioni, Italy).

All samples are tested with TGS TA system for detecting IgG and IgM and, for Group 3, IgG avidity (TGS TA Rubella IgG, Rubella IgM, Rubella IgG Avidity, Technogenetics, Milan, Italy). For Groups 1 and 2, the TGS TA system was used on fresh samples immediately after the results with LIAISON XL test while, for Group 3, TGS TA was used on frozen samples stored on the basis of the results with our protocol.

The TGS TA Rubella IgG and TGS TA Rubella IgM kit employs an indirect two-step immunological method based on the principle of chemiluminescence. The solid phase is coated with a specific antigen or antibody anti h-IgM and the conjugate is or an anti-human IgG antibody or a specific antigen labelled with an acridinium ester derivative. The TGS TA Rubella IgG avidity uses a borate buffer able to prevalently break the binding Antigen (Ag)Antibody $(\mathrm{Ab})$ if the antibody is at low avidity.

The reference values of the different tests are shown in Tables 1 and 2.

With reference to the IgG avidity, low avidity is strongly indicative of an infection occurred in the past 3 months for the ELISA test and 2 months for the TGS TA system; although high avidity does not exclude the possibility of a recent infection, it is strongly indicative of an infection at least 4 months before for
ELISA and 2 months before for TGS TA. The interpretation for medium (ELISA)/moderate (TGS TA) avidity is equivocal and the infection cannot be dated.

\section{Results}

The comparison of the results obtained with the TGS TA and LIAISON XL systems for detecting IgG and IgM gives total concordance of $93.3 \%$ and $89.0 \%$ respectively for IgG and IgM. The overall concordance and that for the different groups is shown in Table 3.

In Group 1 (samples from non-immune women of childbearing age), the samples were classified based on the LIAISON XL IgG values $(\mathrm{IU} / \mathrm{mL})$ :

Sub-group 1a: values $\leq 3 \mathrm{IU} / \mathrm{mL}(\mathrm{n}=64)$

Sub-group 1b: values 4-6 IU/mL $(\mathrm{n}=23)$

Sub-group 1c: values 7-9 IU/mL $(\mathrm{n}=25)$

The concordance between the two systems in the different subgroups is shown in Table 4. In Group 2 (106 samples from pregnant women with past infection or vaccinated), the samples were divided based on the IgG values obtained in IU/mL with LIAISON XL:

Sub-group 2a: values $10 \mathrm{IU} / \mathrm{mL}(\mathrm{n}=11)$

Sub-group $2 \mathrm{~b}$ : values $\geq 11 \mathrm{IU} / \mathrm{mL}(\mathrm{n}=95)$

The concordance between the two systems in the different groups is shown in Table 5 .

The linear regression between TGS TA and LIAISON XL in IgG determination on overall results $(n=267)$ is shown in Figure 1.

In Group 3 (positive or equivocal IgM samples with LIAISON XL), 31 samples were ELFA IgM positive (considered true positive) and 18 ELFA IgM negative (probably non-specific); 33 samples were negative with TGS TA. The results of true positive and non-specific samples with TGS TA are shown in Table 6.

In addition, in Group 3, the $\operatorname{IgG}$ avidity in all samples was determined with both the ELISA and TGS TA methods. The concordance is shown in Table 7.

In particular, of the 17 negative TGS TA IgM samples, but true positive with our protocol (Table 6), 13 had high avidity with both ELISA and TGS TA systems (for the other samples, there were 1 sample with low ELISA/high TGS TA avidity, 2 samples with medium ELISA/high TGS TA avidity and 1 sample with low ELISA/moderate TGS TA avidity).

Table 1. Reference values for the anti-rubella IgG and IgM tests with the LIAISON XL, ELFA and TGS TA systems.

\begin{tabular}{|c|c|c|c|c|c|}
\hline Reference values & $\begin{array}{l}\text { I } \\
\text { LIAISON XL, } \\
\text { IU/mL }\end{array}$ & $\begin{array}{l}\text { TGS TA, } \\
\text { IU/mL }\end{array}$ & $\begin{array}{c}\text { LIAISON XL, } \\
\text { IU/mL }\end{array}$ & $\begin{array}{c}\text { IgM } \\
\text { ELFA } \\
\text { (index) }\end{array}$ & $\begin{array}{l}\text { TGS TA, } \\
\text { IU/mL }\end{array}$ \\
\hline Negative & $<9$ & $<10$ & $<20$ & $<0.80$ & $<10$ \\
\hline Equivocal & $9-11$ & - & $20-25$ & $0.80-1.20$ & - \\
\hline Positive & $>11$ & $\geq 10$ & $>25$ & $>1.20$ & $\geq 10$ \\
\hline
\end{tabular}

Table 2. Reference values for the anti-rubella IgG avidity tests with the ELISA method and TGS TA system.

\begin{tabular}{lcc} 
Reference value & IfG $(\%)$ & TGS TA (index) \\
Low & $<35$ & $<0.20$ \\
Medium/moderate & $35-45$ & $0.20-0.25$ \\
\hline High & $>45$ & $\geq 0.25$ \\
\hline
\end{tabular}


In Group 3, combining LIAISON XL/ELFA and ELISA avidity results, the cases were typified as follows with our protocol: 2 patients with recent infection (ELFA positive IgM and low ELISA avidity); 5 with a non-determined infection (ELFA positive IgM) and medium avidity in ELISA; 24 patients with past infection or vaccinated (ELFA positive IgM and high ELISA avidity); 18 patients with supposed non-specific IgM not confirmed by the ELFA IgM test and with high ELISA avidity.

The concordance between clinical interpretation with our protocol and the TGS TA system is shown in Table 8.

Table 3. Overall concordance in the three groups between LIAISON XL and TGS TA for detecting anti-rubella IgG and IgM antibodies in samples from childbearing-age or pregnant women.

\begin{tabular}{|c|c|c|c|c|}
\hline & & & & \\
\hline & TGS TA neg & TGS TA pos & TGS TA neg & TGS TA pos \\
\hline Overall concor & imples from v & bearing age & & \\
\hline LIAISON XL neg & 97 & 15 & 218 & 0 \\
\hline LIAISON XL pos & 3 & 152 & 29 & 16 \\
\hline LIAISON XL eq & - & - & 4 & 0 \\
\hline Group 1 concol & amples from & romen of chi & & \\
\hline LIAISON XL neg & 97 & 15 & 112 & 0 \\
\hline LIAISON XL pos & 0 & 0 & +1 & 0 \\
\hline Group 2 conco & amples from & en with past & accinated)*** & \\
\hline LIAISON XL neg & 0 & 0 & 106 & 0 \\
\hline LIAISON XL pos & 3 & 103 & 0 & 0 \\
\hline Group 3 conco & les from preg & vith positive & gM to the LIA & \\
\hline LIAISON XL neg & 0 & 0 & 0 & 0 \\
\hline LIAISON XL pos & 0 & 49 & 29 & 16 \\
\hline LIAISON XL eq & - & - & 4 & 0 \\
\hline
\end{tabular}

${ }^{*}$ Overall concordance (excluding equivocal cases): IgG 93.3\%; IgM 89\%. ** Overall concordance: IgG 86.6\%; IgM 100\%. ***Overall concordance: IgG 97.2\%; IgM 100\%. **** Overall concordance (excluding equivocal cases): $\operatorname{IgG} 100 \%$; IgM $35.6 \%$.

Table 4. Concordance between the TGS TA and LIAISON XL systems for anti-rubella IgG class antibodies in non-immune women of childbearing age.

\begin{tabular}{|c|c|c|}
\hline \multicolumn{3}{|c|}{ Sub-group 1a: anti-rubella IgG values $\leq 3 \mathrm{IU} / \mathrm{mL}(\mathrm{n}=64)$} \\
\hline LIAISON XL neg & 64 & 0 \\
\hline LIAISON XL pos & 0 & 0 \\
\hline \multicolumn{3}{|c|}{ Sub-group 1b: anti-rubella IgG values 4-6 IU/mL (n=23) } \\
\hline LIAISON XL neg & 22 & $1^{*}$ \\
\hline LIAISON XL pos & 0 & 0 \\
\hline \multicolumn{3}{|c|}{ Sub-group lc: anti-rubella IgG values 7-9 IU/mL ( $n=25)$} \\
\hline LIAISON XL neg & 11 & $14^{* *}$ \\
\hline LIAISON XL pos & 0 & 0 \\
\hline
\end{tabular}

Concordance: sub-group la, 100\%; sub-group 1b, 95.7\%; sub-group 1c, 44\%.

*Values between $13.5 \mathrm{IU} / \mathrm{mL}$ with TGS TA; **values between 10.6-16.5 IU/mL with TGS TA.

Table 5. Concordance between the TGS TA and LIAISON XL systems for IgG in immune or vaccinated pregnant women.

\begin{tabular}{lcc} 
& IgG & TGS TA pos \\
Sulb-group 2a: anti-rubella IgG values $10 \mathrm{IU} / \mathrm{mL}(\mathrm{n}=11) *$ & TGS TA neg & 0 \\
LIAISON XL neg & 0 & 8 \\
LIAISON XL pos & 3 & 0 \\
\hline Sulb-group 2b: anti-rubella IgG values $\geq 11 \mathrm{IU} / \mathrm{mL}(\mathrm{n}=95) * *$ & 0 & 95 \\
LIAISON XL neg & 0 & \\
LIAISON XL pos & 0 & \\
\hline
\end{tabular}

${ }^{*}$ Concordance $72.7 \% ;{ }^{* *}$ concordance $100 \%$. 
In Group 1 (112 non-immune women of childbearing age), comparison of the clinical diagnosis obtained with our protocol and that with the TGS TA system, showed $86.6 \%$ concordance where the TGS TA system highlighted $15(13.4 \%)$ with low titre IgG (values between 10.6 and $16.7 \mathrm{IU} / \mathrm{mL}$ ); the same subjects all had values of 6-9 IU/mL with LIAISON XL.

In Group 2 (106 immune women), concordance was $97.2 \%$ the TGS TA test only highlighted 3 non-immune $(2.8 \%)$ with value close to the cut-off $(9.7 \mathrm{IU} / \mathrm{mL})$ in one case. The other two values were 5.6 and $7.1 \mathrm{IU} / \mathrm{mL}$. All three values were at the cut-off (10 $\mathrm{IU} / \mathrm{mL}$ ) in the LIAISON XL test.

Finally, considering all the three groups under examination, the overall clinical concordance is shown in Table 9.

\section{Discussion and Conclusions}

The concordance between the LIAISON XL and TGS TA systems for IgG seems to be generally good even if with variations according to the 3 groups. The TGS TA test seems to be particularly sensitive in indicating the immunity of vaccinated people with low levels of IgG. The lack of standardisation and the introduction of rubella vaccination programmes explain the differences in the interpretation of the results for low values of $\mathrm{IgG}$, around the cutoff $(5,6)$. One consequence of the rubella vaccination programme is an overall reduction in the levels of rubella virus IgG in vaccinated individuals compared with those acquiring immunity through natural infection $(5,6,9)$. This causes difficulties in clinical interpretation of immune or non-immune, especially if the grey zone is not given $(5,6)$. The TGS TA system does not have a grey area around the cut-off unlike the LIAISON XL and ELFA and the calculation of the concordance suffers from this limitation.

The LIAISON XL and TGS TA tests for IgM correlate well in the first two groups, while the correlation is low in the third group, but this is also affected by patients with non-specific IgM in this group. The correlation is higher if very positive are evaluated separately from non-specific samples. The TGS TA system indicates the majority of non-specific samples as negative with our protocol, suggesting a good specificity. Further, the TGS TA system indicates as positive less than half of the samples resulting true positive with our protocol but the majority of these cases are past infections and the positivity of IgM may confound.

The limit of the study design is that it suffers from a lack of cases of recent rubella infection in our centre. However, this is due to the introduction of the vaccination campaigns which have made rare observation of acute rubella.

The comparison data demonstrate good overall clinical concordance. The TGS TA system clearly indicates individuals considered non-specific or with past infection; although it indicates an additional case of recent infection, it misses one with respect to our protocol. Clinical discordance may also depend on the different method of dating the infection of the avidity tests of the two systems. In the ELISA test, low avidity is strongly indicative of an infection in the past 3 months while, in the TGS TA test this indicates an infection in the past 2 months; although not excluding the

\section{RUBELLA IgG: TGS TA vs LIAISON XL}

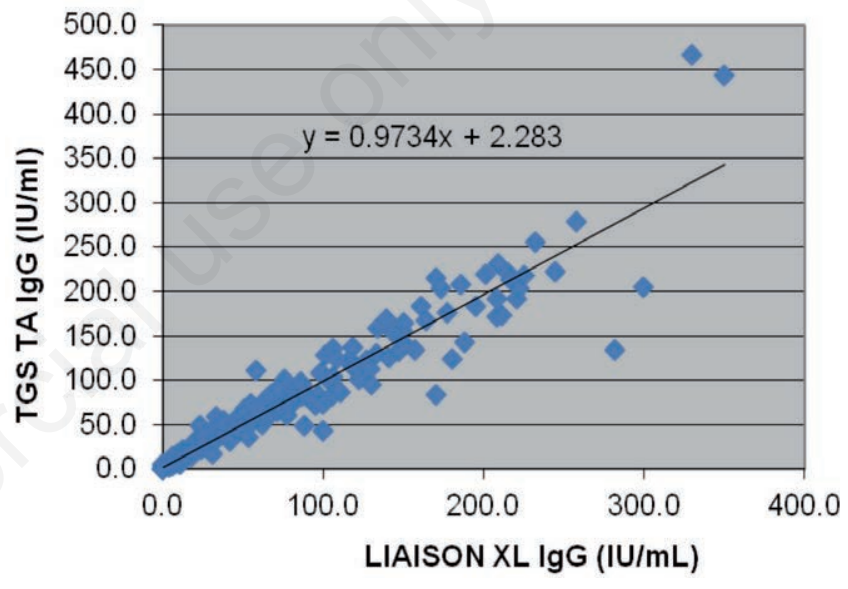

$\mathrm{R}^{2}=0.973$

Figure 1. Linear regression between TGS TA and LIAISON XL of the anti-rubella IgG (IU/mL).

Table 6. Comparison between LIAISON XL/ELFA with TGS TA IgM tests.

\begin{tabular}{lccccc} 
Our protocol & \multicolumn{2}{c}{ TGS TA } & Total & \multicolumn{2}{c}{ Concordance, \% } \\
& Positive & Negative & 31 & 45.2 \\
LIAISON XL/ELFA both positive or equivocal (true positive) & 14 & 17 & 18 & 88.9 \\
LIAISON XL positive/ELFA negative (nonspecific) & 2 & 16 & 49 & 61.2 \\
\hline Total & 16 & 33 & 3 \\
\hline
\end{tabular}

Table 7. Concordance between the ELISA and TGS TA IgG avidity tests in subjects with positive or equivocal IgM in the LIAISON XL.

\begin{tabular}{lcccc} 
& \multicolumn{2}{c}{ TGS TA avidity } & High & Total \\
ELISA avidity & Low & & \\
Low & & & 1 & 2 \\
Medium & 0 & 0 & 4 & 5 \\
High & 1 & 0 & 42 & 42 \\
Total & 0 & 1 & 47 & 49 \\
\hline
\end{tabular}

Concordance: $85.7 \%$. 
Table 8. Comparison between the clinical interpretations given with the LIAISON/ELFA/ELISA protocol and TGS TA.

\begin{tabular}{lcccc} 
UIAISON/LFA & $\begin{array}{c}\text { Probable } \\
\text { DIESSE protocol }\end{array}$ & $\begin{array}{c}\text { Thdeterminate } \\
\text { infection }\end{array}$ & $\begin{array}{c}\text { Non-recent } \\
\text { infection/vaccinated }\end{array}$ \\
Probable recent infection & 0 & 1 & 1 & 2 \\
Undeterminate infection & 1 & 0 & 4 & 4 \\
\hline Non-recent infection/vaccinated & 0 & 0 & 42 & 42 \\
Total & 1 & 1 & 47 & 49 \\
\hline
\end{tabular}

Concordance: $85.7 \%$.

Table 9. Clinical concordance between the LIAISON/ELFA/DIESSE protocol and the TGS TA system considering all three groups under examination.

\begin{tabular}{|c|c|c|c|c|c|}
\hline $\begin{array}{l}\text { LIAISON/ELFA } \\
\text { DIESSE protocol }\end{array}$ & $\begin{array}{l}\text { Probable } \\
\text { recent infection }\end{array}$ & $\begin{array}{c}\text { Undeterminate } \\
\text { infection }\end{array}$ & $\begin{array}{c}\text { TGS TA system } \\
\text { Non-recent } \\
\text { infection/vaccinated }\end{array}$ & Non-immune & Total \\
\hline Probable recent infection & 0 & 1 & 1 & 0 & 2 \\
\hline Undeterminate infection & 1 & 0 & 4 & 0 & 5 \\
\hline Non-recent infection/vaccinated & 0 & 0 & 160 & 0 & 160 \\
\hline Non-immune & 0 & 0 & 0 & 100 & 100 \\
\hline Total & 1 & 1 & 165 & 100 & 267 \\
\hline
\end{tabular}

Total concordance: $97.4 \%$.

possibility of a recent infection, high avidity in ELISA is strongly indicative of an infection at least 4 months before while for TGS TA, the infection occurred more than 2 months before. As a result, most of the infections indicated as undetermined with our protocol are considered as past with TGS TA.

\section{References}

1. Amendola A, Bubba L, Piralla A, et al. Surveillance and vaccination coverage of measles and rubella in Northern Italy. Hum Vaccin Immunother 2015;11:206-13.

2. Cooper LZ, Altford CA, Rubella. In: Infectious diseases of the fetus and newborn infant. Remington JS, Klein JO, Baker C, Wilson C, eds. Philadelphia: Elsevier Saunders; 2006. pp 893-926.

3. De Paschale M, Manco MT, Paganini A, et al. Rubella antibody screening during pregnancy in an urban area of Northern Italy. Infect Dis Rep 2012;4:e17.

4. De Santis M, Cavaliere AF, Straface G, Caruso A. Rubella infection in pregnancy. Reprod Toxicol 2006;21:390-8.

5. Dimech W, Grangeot-Keros L, Vauloup-Fellos C. Standardization of assays that detect anti-rubella virus $\mathrm{IgG}$ antibodies. Clin Microbiol 2016:163-74.

6. Francis BH, Thomas AK, McCarty CA The impact of rubella immunization on serological status of women childbearing age: a retrospective longitudinal study in Melbourne, Australia. Am J Public Health 2003;93:1274-6.

7. Giambi C, Filia A, Rota MC, et al. Congenital rubella still a public health problem in Italy: analysis of national surveillance data from 2005 to 2013. Euro Surveill 2015;20:21103.

8. Giambi C, Montaño-Remacha C, Celentano LP, et al. Surveillance of congenital rubella and rubella infections in pregnancy in EU/EEA countries, 2012: current status and future perspective to monitor elimination. Vaccine $2015 ; 33$ :4929-37.

9. Le Baron C, Forghani B, Matter L, et al. Persistence of rubella antibodies after 2 doses of measles-mumps-rubella vaccine. J Infect Dis 2009;200:888-9.

10. Mellinger AK, Cragan JD, Atkinson WL, et al. High incidence of congenital rubella syndrome after a rubella outbreak. Pediatr Infect Dis J 1995;14:573-8.

11. State Regional Conference. Agreement between the central Government, Regions and Autonomous Provinces of Trento and Bolzano regarding the National Measles and Congenital Rubella Elimination Plan 2003-2007. 13 November 2003.

12. World Health Organisation. Report of a meeting on preventing congenital rubella syndrome: immunization strategies, surveillance needs, Geneva, 12-14 January 2000. Geneva: World Health Organisation; 2000. 